\title{
dspace.vutbr.cz
}

\section{Practical Design of Fractional-Order Resonator for Application in the Multiphase Oscillator}

\author{
ŠOTNER, R.; JEŘÁBEK, J.; LANGHAMMER, L.; KARTCI, A.; HERENCSÁR, N.; METIN, B.
}

Proceedings of the 2020 27th IEEE International Conference on Electronics, Circuits and Systems (ICECS)

elSBN: 978-1-7281-6044-3

DOI: https://doi.org/10.1109/ICECS49266.2020.9294799

Accepted manuscript

C2020 IEEE. Personal use of this material is permitted. Permission from IEEE must be obtained for all other uses, in any current or future media, including reprinting/republishing this material for advertising or promotional purposes, creating new collective works, for resale or redistribution to servers or lists, or reuse of any copyrighted component of this work in other works. ŠOTNER, R.; JEŘÁBEK, J.; LANGHAMMER, L.; KARTCI, A.; HERENCSÁR, N.; METIN, B.

"Practical Design of Fractional-Order Resonator for Application in the Multiphase Oscillator", 27th IEEE International Conference on Electronics, Circuits and Systems (ICECS), 2020.

DOI: 10.1109/ICECS49266.2020. 9294799. Final version is available at

https://ieeexplore.ieee.org/document/9294799 


\title{
Practical Design of Fractional-Order Resonator for Application in the Multiphase Oscillator
}

\author{
Roman Sotner*, Jan Jerabek ${ }^{*}$, Lukas Langhammer ${ }^{*}$, Aslihan Kartci ${ }^{*}$, Norbert Herensar ${ }^{*}$, Bilgin Metin ${ }^{\dagger}$ \\ Email: \{sotner, jerabekj, langhammer, kartci, herencsn\}@feec.vutbr.cz, bilgin.metin@boun.edu.tr \\ *Faculty of Electrical Engineering and Communication, Brno University of Technology, Brno, Czech Republic \\ †Department of Management Information Systems, Bogazici University, Istanbul, Turkey
}

\begin{abstract}
This contribution introduces a new simple electronically adjustable resonator for implementation in design of the fractional-order multiphase oscillator created with parallel connection of this resonator and negative resistor. The circuit may operate in single-ended or differential/pseudodifferential mode using integer and fractional-order capacitor. Design example is provided for generation of signals with ideal phase shifts equal to $22.5,157.5$ and 180 degrees in the operating frequency band of $47 \mathrm{kHz}-275 \mathrm{kHz}$ with simple tuning of a single parameter. The features of the circuit were verified by PSpice simulations using suitable models and building blocks. Results confirmed expected behavior.
\end{abstract}

Keywords-Constant phase element, electronic tuning, fractional-order, multiphase oscillator, resonator.

\section{INTRODUCTION}

Standard synthesis and design of fractional-order oscillators supposes very uncomfortable expression for oscillation condition and oscillation frequency. This type of equation is not very suitable for direct hand calculations [1], [2]. Nevertheless, recent years brought many interesting concepts of fractional-order oscillators that were studied in [1]-[15]. Table 1 provides their brief overview. However, their design is not simple and many features (electronic tunability and relation of phase shifts when oscillation frequency is tuned, etc.) are not analyzed. As we can see, also number of existing multiphase solutions (generating three and more output signals) is not very high [1], [10], [12], [13]. The standard analysis of these circuits targets on the study of parameters of characteristic equation, except [10] and [15]. It seems that tunability of oscillation frequency in these types of oscillators received minimal attention of researchers in recent works. There are only several papers [11]-[13] where variation of the order of fractional-order element (abbreviated as FOE), known also as the constant phase element (CPE), has been studied theoretically for fulfilment of oscillation condition or oscillation frequency tuning. Unfortunately, this is not very practical way when these oscillators should be tuned immediately and electronically. Paper [10] introduces the verification of the tunability by variation of passive element (resistor). Unfortunately, many further beneficial features (phase and amplitude relations between produced waveforms, suitability for usage in differential mode, suitability for multiphase operation, simplified design, way for stabilization of amplitudes, etc.) are not available, except the first partial attempt presented in [15].

Our approach provides a different view on the circuit based on analysis of very complex characteristic equations (complex even in ideal form - without taking parasitic

This article is based upon work from COST Action CA15225, a network supported by COST (European Cooperation in Science and Technology). Research described in this paper was financed by the Ministry of Education, Youth and Sports under grant LTC18022 of INTERCOST program. influences into account). Our concept analyses the oscillator as parallel connection of resonator (based on an impedance converter/gyrator) and negative resistor. Of course, we cannot generalize this approach for any topology, but it still provides interesting results. This approach allows us to study features of the resonator independently on the parameters of the rest of the oscillating structure (negative resistor serves only for undamped operation of the resonator). It offers straightforward way for understanding operations in the circuit and logical derivation of all-important design equations in practically applicable form.

TABLE I. COMPARISON OF RECENT OSCILLATOR DESIGNS USING FRACTIONAL-ORDER DEVICES

\begin{tabular}{|c|c|c|c|c|c|c|c|c|}
\hline 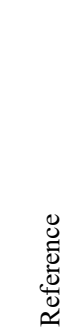 & 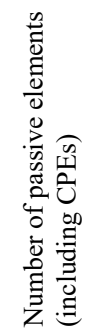 & 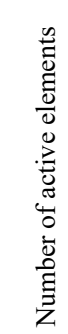 & 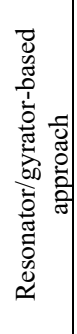 & 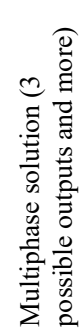 & 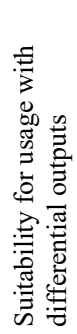 & 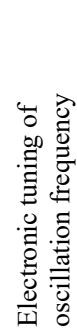 & 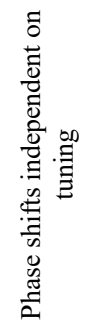 & 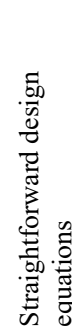 \\
\hline$[1]$ & $3-7$ & 1 & No & Yes & No & N/A & N/A & No \\
\hline [2] & $>7$ & $2-3$ & Yes & No & No & N/A & N/A & No \\
\hline [3] & 3 & 4 & $\mathrm{No}$ & No & No & $\mathrm{N} / \mathrm{A}$ & N/A & No \\
\hline [4] & 3 & $1 *$ & No & No & No & N/A & N/A & No \\
\hline [5] & 6 & 1 & No & No & No & N/A & N/A & No \\
\hline [6] & 4 & $6^{*}$ & No & No & No & N/A & N/A & No \\
\hline [7] & 4 & 1 & No & No & No & $\mathrm{N} / \mathrm{A}$ & N/A & $\mathrm{No}$ \\
\hline [8] & 6 & 2 & No & No & No & N/A & N/A & No \\
\hline [9] & $5-7$ & 1 & Yes & No & No & $\mathrm{N} / \mathrm{A}$ & N/A & No \\
\hline$[10]$ & 4 & 2 & No & Yes & No & Yes & Yes & No \\
\hline [11] & 4-7 & $1-2$ & No & No & No & N/A & N/A & No \\
\hline [12] & 8 & 3 & $\mathrm{No}$ & Yes & No & N/A & N/A & No \\
\hline [13] & 8 & $3-4$ & $\mathrm{No}$ & Yes & No & $\mathrm{N} / \mathrm{A}$ & $\mathrm{N} / \mathrm{A}$ & No \\
\hline [14] & 5 & 1 & No & N/A & No & N/A & N/A & No \\
\hline$[15]$ & 2 & 1 & Yes & No & No & Yes & Yes & Yes \\
\hline Fig. 3 & $5(7)^{+}$ & $5(6)^{+}$ & Yes & Yes & Yes & Yes & Yes & Yes \\
\hline
\end{tabular}

${ }^{*}$ CMOS cells used (number of functional parts/devices); ${ }^{+} 7$ passive and 6 active devices required when additional resistors used in nodes and voltage buffer with differential output considered; N/A - information not available, not tested

\section{Proposed Structure of the Resonator}

The proposed structure of the resonator used in [15] includes quite interesting connection of active devices and two grounded CPEs. However, more generalized solution can be proposed and is presented in this paper including possibility of implementation with differential/pseudodifferential [16] outputs (Fig. 1). The ideal resonator includes two passive elements (inductance and capacitance of order between 0 and 1). 


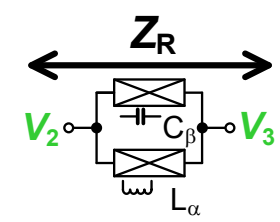

Fig. 1. Floating impedance resonator using CPEs.

The solution is highlighted in right part of Fig. 2. The functionality of active devices is very simple. The electronically controllable current conveyor of second generation (ECCII-) [17], [18] performs the following operations: $\quad V_{\mathrm{X}}=V_{\mathrm{Y}}, \quad I_{\mathrm{Y}}=0, \quad I_{\mathrm{Z}}=-B \cdot I_{\mathrm{X}}, \quad$ and voltage differencing differential difference amplifier (VDDDA) [19], [20] used with full negative feedback brings simple summation/subtracting operation in form: $V_{\text {out }}=\left(V_{+}+V_{+}-V_{-}\right)$.

The overall impedance of the resonator can be expressed as:

$$
Z_{R}(s)=\frac{V_{\text {in }}}{I_{\text {in }}}=\frac{s^{\alpha} L_{\alpha}}{s^{\alpha+\beta} L_{\alpha} C_{\beta}+1}=\frac{s^{\alpha} C_{\alpha} R_{1} \frac{R_{2}}{B_{1}}}{s^{\alpha+\beta} C_{\alpha} C_{\beta} R_{1} \frac{R_{2}}{B_{1}}+1} .
$$

The equivalent inductance has its value adjustable by current gain $B_{1}$ of the ECCII [17], [18] in the form of $L_{\alpha}=C_{\alpha} R_{1} R_{2} / B_{1}$. It means that $L_{\alpha}$ is transformed from $C_{\alpha}$ (fractional-order capacity - indicated as $Z_{\mathrm{CPE} 1}$ in Fig. 2) by simple impedance converter core of the resonator (all active and passive parts except $Z_{\mathrm{CPE} 2}$ ). Note that obtainment of the following expressions requires support of some mathematical tool for solution and simplification of symbolical expressions. Connection with floating $Z_{\mathrm{CPE} 2}$ (fractional-order capacity) forms ideal behavior described by (1). The impedance $Z_{\mathrm{R}}(\mathrm{s})$ can be expressed also as:

$$
Z_{R}(j \omega)=\frac{\omega^{\alpha}\left[\cos \left(\alpha \frac{\pi}{2}\right)+j \sin \left(\alpha \frac{\pi}{2}\right)\right] C_{\alpha} R_{1} \frac{R_{2}}{B_{1}}}{\omega^{\alpha+\beta}\left[\cos \left(\frac{(\alpha+\beta) \pi}{2}\right)+j \sin \left(\frac{(\alpha+\beta) \pi}{2}\right)\right] C_{\alpha} C_{\beta} R_{1} \frac{R_{2}}{B_{1}}+1}
$$

All oscillators operate when their closed-loop transfer reaches sufficiently high gain $(0 \mathrm{~dB})$ as well as the phase response crosses $0^{\circ}$ [21]. The resonant frequency of the resonator $\omega_{0}$ can be found easily from: $\varphi\left(\omega_{0}\right)=\tan ^{-1}\left[\operatorname{num}\left(Z_{\mathrm{R}}\left(j \omega_{0}\right)\right)\right]-\tan ^{-1}\left[\operatorname{den}\left(Z_{\mathrm{R}}\left(j \omega_{0}\right)\right)\right]$, because the phase response crosses (equals) $0^{\circ}$ when this value is reached, i.e.: $\tan ^{-1}\left[\operatorname{num}\left(Z_{\mathrm{R}}(s)\right)\right]=\tan ^{-1}\left[\operatorname{den}\left(Z_{\mathrm{R}}(s)\right)\right]$, that can be substituted by:

$$
\tan ^{-1}\left[\frac{\sin \left(\alpha \frac{\pi}{2}\right)}{\cos \left(\alpha \frac{\pi}{2}\right)}\right]=\tan ^{-1}\left[\frac{\omega^{\alpha+\beta} \sin \left((\alpha+\beta) \frac{\pi}{2}\right) C_{\alpha} C_{\beta} R_{1} \frac{R_{2}}{B_{1}}}{\omega^{\alpha+\beta} \cos \left((\alpha+\beta) \frac{\pi}{2}\right) C_{\alpha} C_{\beta} R_{1} \frac{R_{2}}{B_{1}}+1}\right],
$$

and the frequency while crossing $0^{\circ}$ is in the form of:

$$
\omega_{0}=\left[\frac{\frac{B_{1}}{C_{\alpha} C_{\beta} R_{1} R_{2}} \sin \left(\alpha \frac{\pi}{2}\right)}{\cos \left(\alpha \frac{\pi}{2}\right) \cdot \sin \left((\alpha+\beta) \frac{\pi}{2}\right)-\sin \left(\alpha \frac{\pi}{2}\right) \cdot \cos \left((\alpha+\beta) \frac{\pi}{2}\right)}\right]^{\frac{1}{\alpha+\beta}}
$$

The magnitude of the impedance at the frequency $\omega_{0}$ is found as:

$$
\left|Z_{R}\left(\omega=\omega_{0}\right)\right|=\frac{C_{\alpha} R_{1} \frac{R_{2}}{B_{1}}\left(\frac{B_{1} \sin \left(\alpha \frac{\pi}{2}\right)}{C_{\alpha} C_{\beta} R_{1} R_{2} \sin \left(\beta \frac{\pi}{2}\right)}\right)^{\frac{\alpha}{\alpha+\beta}}}{\sqrt{\left(\frac{\sin \left(\alpha \frac{\pi}{2}\right)}{\sin \left(\beta \frac{\pi}{2}\right)}\right)^{2}+2 \cos \left((\alpha+\beta) \frac{\pi}{2}\right)\left(\frac{\sin \left(\alpha \frac{\pi}{2}\right)}{\sin \left(\beta \frac{\pi}{2}\right)}\right)+1}}
$$

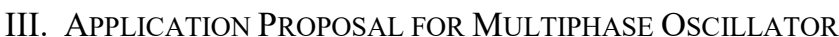

Simple completion of the topology in Fig. 1 by controlled negative resistance fulfills all requirements for signal generation within the structure, because negative resistance eliminates losses in the structure when properly set, see Fig. 2. This in fact supports beneficial features discussed in further text. The circuit in Fig. 2 is suitable for differential/pseudo-differential generation $\left(V_{2}-V_{3}\right.$ and $V_{1}-V_{1}$ ) after simple modification (additional special voltage buffer producing signals of both polarities - dashed lines).

The oscillation frequency of this oscillator is given by (4) and amplitude condition fulfillment bases on: $R_{\text {neg }} \cong\left|Z_{\mathrm{R}}\left(\omega_{0}\right)\right|$ $\cong R_{3} / B_{2} \cong R_{3} / V_{\mathrm{SETB} 2}$. It means that design and setting of the oscillator by presented approach is much more simpler than approaches requiring comprehensive steps and complex mathematical background shown in [1]-[14], where full oscillation condition is analyzed and clear dependences of oscillation frequency as well as oscillation condition on circuit parameters are unsuitable for fast and simple design and easy electronic controllability. The voltage transfer between nodes $V_{1}$ and $V_{2}$ in green color yields:

$$
\left.\frac{V_{1}}{V_{2}}\right|_{\omega=\omega_{0}}=\frac{-1}{s^{\alpha} C_{\alpha} R_{1}}=\frac{-e^{j \cdot \tan ^{-1}\left[\frac{\sin \left(\alpha \frac{\pi}{2}\right)}{\cos \left(\alpha \frac{\pi}{2}\right)}\right]}}{C_{\alpha} R_{1}\left[\frac{\sin \left(\alpha \frac{\pi}{2}\right)}{C_{\alpha} C_{\beta} R_{1} \frac{R_{2}}{B_{1}} \sin \left(\beta \frac{\pi}{2}\right)}\right]^{\frac{\alpha}{\alpha+\beta}}}
$$

The equation (6) gives relation between voltage ratio and phase shift of generated signals that is equal to:

$$
\varphi_{1-2}=180-\tan ^{-1}\left[\frac{\sin \left(\alpha \frac{\pi}{2}\right)}{\cos \left(\alpha \frac{\pi}{2}\right)}\right] \Rightarrow \varphi_{1-2}=180-\alpha \frac{\pi}{2}
$$

Independence of the phase shift on $\beta$ (clear from (7)) is the most interesting finding of this analysis. The similar relation for the phase shift as (6) is also valid for nodes $V_{1}$ and $V_{3:} \varphi_{1-}$ ${ }_{3}=-\alpha \cdot \pi / 2$ (the amplitude ratio has the same form). Phase and voltage relation of voltages in nodes $V_{2}$ and $V_{3}$ is simple inversion $\left(180^{\circ}\right.$ phase shift). However, the implementation of both capacitors in fractional form (CPEs) is useful for obtainment of various dependences of oscillation frequency on time constants (parameters influencing time constants) because both orders impact power of expression for frequency. 


\section{DESIGN AND ANALYSIS OF THE RESONATOR}

We selected the following parameters: $\alpha=0.25$, $C_{\alpha}=220 \mu \mathrm{F} / \mathrm{sec}^{3 / 4}, \Delta \varphi_{\alpha}= \pm 2^{\circ}$ designed for the frequency range of $30 \mathrm{~Hz}$ and $10 \mathrm{MHz} ; \beta=1, C_{\beta}=1 \mathrm{nF}, R_{1}=R_{2}=$ $220 \Omega, B_{1}=1$ (obtained by $V_{\mathrm{SETB} 1}=1 \mathrm{~V}$, see datasheet of EL2082). Figure 3 shows impedance plots of the CPE. The resonator impedance characteristic is shown for variation of $B$ parameter $(0.2,0.5,1,2)$ in Fig. 4 . The tunability expects range between $10 \mathrm{kHz}$ and $1 \mathrm{MHz}$ (respecting active devices). Passive RC CPE solution including designed values (based on approaches presented in [22], [23]) is given in Fig. 5. Practical examples of the design and Matlab algorithm are available in [24], [25], for instance.

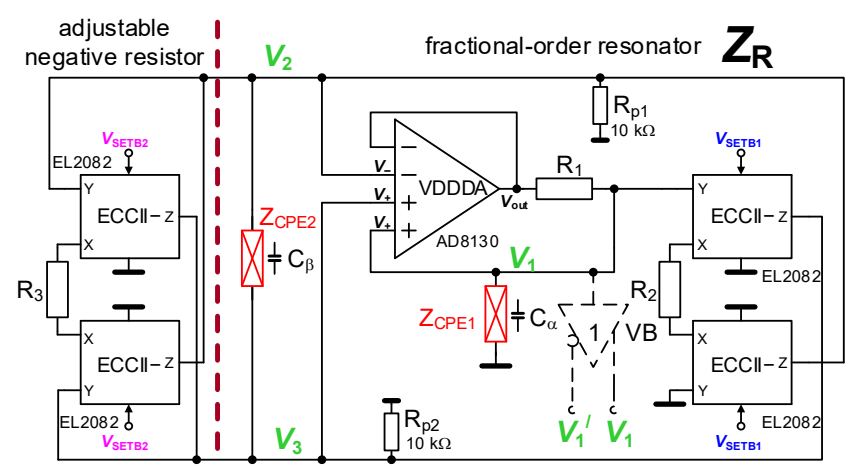

Fig. 2. Proposed application - multiphase oscillator based on resonator and negative resistor.

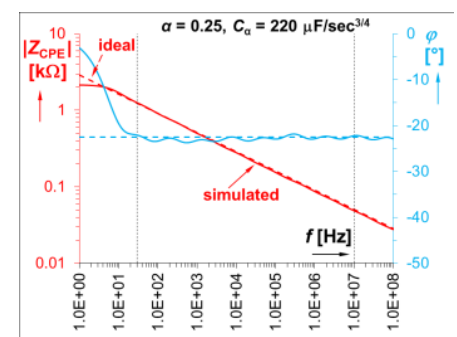

Fig. 3. Impedance plot of CPE used in further design.

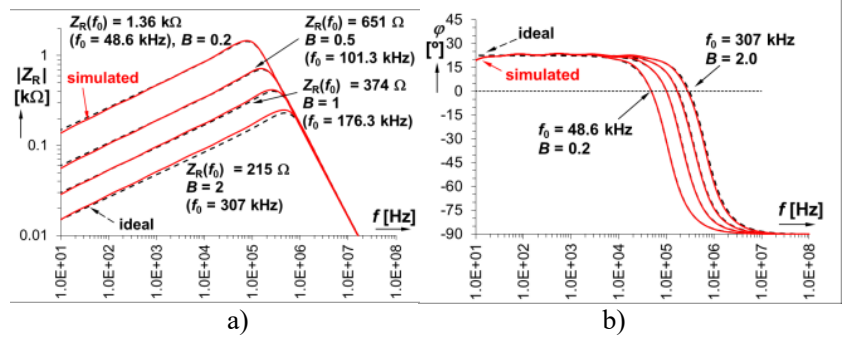

Fig. 4. Impedance plots of the resonator for selected values of $B(0.2,0.5$, $1,2)$ - simulation results: a) magnitudes, b) phase responses.

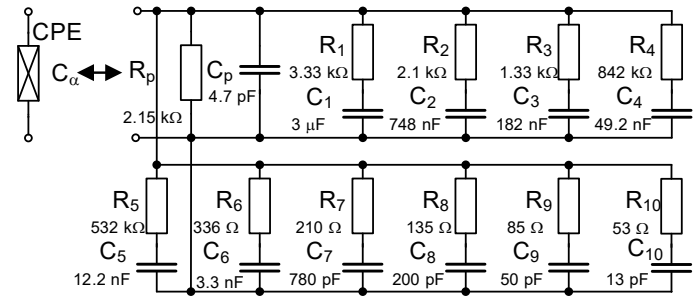

Fig. 5. The RC realization of CPE $\left(\alpha=0.25, C_{\alpha}=220 \mu \mathrm{F} / \mathrm{sec}^{3 / 4}\right)$ used in resonator structure as $C_{\alpha}$.

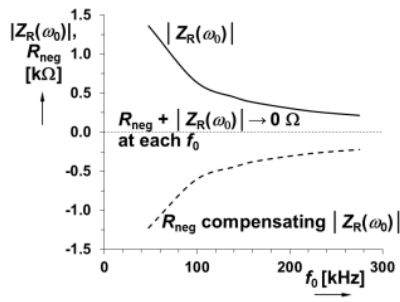

a)

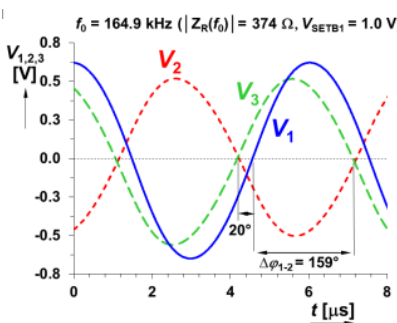

b)
Fig. 6. Exemplary results: a) magnitude impedance plots of resonator and negative resistor when $f_{0}$ varied, b) selected example of output waveforms of the oscillator.

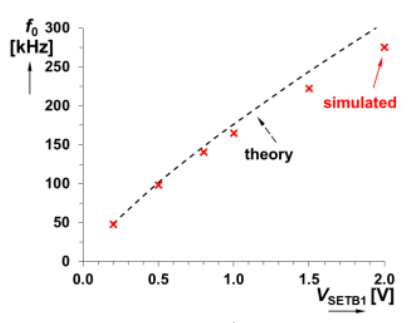

a)

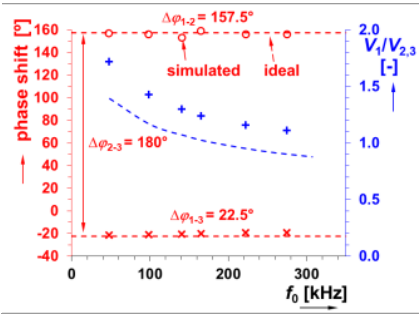

b)
Fig. 7. Dependence of: a) oscillation frequency on driving parameter $\left.V_{\mathrm{SETB} 1}, \mathrm{~b}\right)$ phase shifts between nodes $V_{1-3}$ and amplitude ratios on tuning procedure.

\section{VERIFICATION OF THE OSCILLATOR}

We used previously noted features of the resonator for simple design of the multiphase oscillator generating output voltages in three independent nodes of the structure. The passive elements and other parameters remain identical to resonator design. Resistors values $(10 \mathrm{k} \Omega)$ sufficiently higher than rest of resistors in the circuit in Fig. 2 were selected for $R_{\mathrm{p} 1,2}$ (avoidance of impact of DC offset, reference to analog ground, with insignificant impact on other features). We studied behavior of the circuit for process of electronic tuning by variation of $V_{\text {SETB1 }}$ between 0.2 and $2.0 \mathrm{~V}$. As we know, the magnitude $\left|Z_{\mathrm{R}}\left(\omega_{0}\right)\right|$ decreases with oscillation frequency $f_{0}$. Therefore, the negative resistor $\left(R_{\text {neg }}\right)$ must compensate these changes as shown in Fig. 6(a). The value of $R_{\text {neg }}$ can be driven from specific circuit for amplitude stabilization in practice (as shown in [15]). The tested theoretical range of $f_{0}$ was between $48.6 \mathrm{kHz}$ and $307 \mathrm{kHz}$ (Fig. 6 and Fig. 7). The PSpice simulations yield tunability between $47.6 \mathrm{kHz}$ and $275 \mathrm{kHz}$. The obtained phase shifts are studied in Fig. 7(a). Based on equation (6), we expect impact of $f_{0}$ tuning on ratio between generated amplitudes $\left(V_{1} / V_{2,3}\right)$. However, the phase shift remains unchanged during the tuning process. The example of time domain responses for $f_{0}=164.5 \mathrm{kHz}\left(V_{\mathrm{SETB} 1}=1 \mathrm{~V}\right)$ is shown in Fig. $6(\mathrm{~b})$.

Many other simulations and analyses were performed, however cannot be presented in graphical form in this paper because of limited space. Therefore, at least some numerical results are presented in this paragraph. Output amplitudes reach levels between 740 and $1020 \mathrm{mV}_{\text {P-P }}\left(V_{2}\right)$, between 780 and $1080 \mathrm{mV}_{\text {P-P }}\left(V_{3}\right)$ and between 940 and $1420 \mathrm{mV}_{\text {P-P }}\left(V_{1}\right)$ in observed range of $f_{0}$ tuning $(47.6 \mathrm{kHz} \rightarrow 275 \mathrm{kHz})$. The total harmonic distortion was found between 2-3\%. The error between theoretical and simulated phase shift $\left(\varphi_{1-2}\right)$ was found between 4 and $15 \%$ and between 0.3 and $3 \%$ for $\varphi_{1-3}$. The error of value $f_{0}$ reaches values between 2 and $10 \%$ in observed range. The error of amplitude ratio has systematical character about $23-26 \%$ against theoretical dependence. 
These errors are acceptable when considering all inaccuracies, operation in hundreds of $\mathrm{kHz}$ and complexity of the circuitry. Of course, some of these errors could be compensated, if necessary.

\section{CONCLUSION}

It seems that combination of integer- and fractional-order capacitors in specific structure of the oscillator offer different features than oscillators using two identical CPEs [15]. However, the phase shift between generated signals seems to be given by fractional-order device only. We tested designed electronically tunable multiphase oscillator employing special resonator circuit and negative resistor in $f_{0}$ range $47.6 \rightarrow 275 \mathrm{kHz}$ and preserving phase shifts between generated amplitudes for combination elements having integer- and fractional-order character. Moreover, this circuit can be easily used as differential/pseudo-differential source of signal. There are still many unanswered questions. Future research will target on study of structure using accumulating elements of different fractional order $(\alpha, \beta)$ and practical features of the circuit in tuning operation (phase shift, ratio of generated amplitudes as well as proposal of suitable systems for amplitude stabilization). Some modifications of the concept should be considered and studied for obtainment of more output phases as available in [26] for example. However, these multiphase solutions use different concepts (topologies using arbitrary number of lossy sections in closed loop) offering more than two output phases.

\section{REFERENCES}

[1] A. G. Radwan, A. S. Elwakil, A. M. Soliman, "Fractional-order sinusoidal oscillators: design procedure and practical examples," IEEE Trans. on Circuits and Systems I: Regular Papers, vol. 55, no. 7, pp. 2051-2063, 2008.

[2] L. A. Said, A. G. Radwan, A. H. Madian, and A. M. Soliman, "Twoport two impedances fractional order oscillators," Microelectronics Journal, vol. 55, no. 9, pp. 40-52, 2016.

[3] A. Kartci, N. Herencsar, J. Koton, L. Brancik, K. Vrba, G. Tsirimokou, and C. Psychalinos, "Fractional-order oscillator design using unity-gain voltage buffers and OTAs," In Proc. of the 2017 IEEE 60th International Midwest Symposium on Circuits and Systems (MWSCAS), Boston, USA, 2017, pp. 555-558.

[4] A. Kartci, N. Herencsar, J. Koton, and C. Psychalinos, "Compact MOS-RC Voltage-Mode Fractional-Order Oscillator Design," In Proc. of the 2017 23rd European Conference on Circuit Theory and Design (ECCTD), Catania, Italy, 2017, pp. 1-4.

[5] A. Kartci, A. Agambayev, A. H. Hassan, H. Bagci, and K. N. Salama, "Experimental Verification of a Fractional-Order Wien Oscillator Built Using Solid-State Capacitors," In Proc. of the 2018 IEEE 61st International Midwest Symposium on Circuits and Systems (MWSCAS), Windsor, ON, Canada, 2018, pp. 544-545.

[6] A. Kartci, N. Herencsar, L. Brancik, and K. N. Salama, "CMOS-RC Colpitts Oscillator Design Using Floating Fractional-Order Inductance Simulator," In Proc. of the 2018 IEEE 61st international Midwest Symposium on Circuits and Systems (MWSCAS), Windsor, ON, Canada, 2018, pp. 905-908.

[7] A. Kartci, N. Herencsar, J. Dvorak, K. Vrba, "VDIBA-Based Fractional-Order Oscillator Design", In Proc. of the 201942 nd International Conference on Telecommunications and Signal Processing (TSP), Budapest, Hungary, 2019, pp. 744-747.

[8] A. Kartci and L. Brancik, "CFOA-based fractional-order oscillator design and analysis with NILT method," In Proc. of 2017 27th International Conference Radioelektronika (RADIOELEKTRONIKA), Brno, Czech Republic, 2017, pp. 1-4.

[9] L. A. Said, A. G. Radwan, A. H. Madian, A. M. Soliman, "Fractional Order Oscillator Design Based on Two-Port Network," Circuits, Systems, and Signal Processing, vol. 35, no. 9, pp. 3086-3112, 2016.
[10] D. Kubanek, F. Khateb, G. Tsirimokou, C. Psychalinos, "Practical Design and Evaluation of Fractional-Order Oscillator Using Differential Voltage Current Conveyors," Circuits, Systems, and Signal Processing, vol. 35, no. 6, pp. 2003-2016, 2016.

[11] L. A. Said, A. G. Radwan, A. H. Madian, A. M. Soliman, "Fractional order oscillators based on operational transresistance amplifiers," AEU - International Journal of Electronics and Communications, vol. 69, no. 7, pp. 988-1003, 2015.

[12] L. A. Said, A. G. Radwan, A. H. Madian, A. M. Soliman, "Three Fractional-Order-Capacitors-Based Oscillators with Controllable Phase and Frequency," Journal of Circuits, Systems and Computers, vol. 26, no. 10, pp. 1750160-1750178, 2017.

[13] M. Fouda, A. Soltan, A. G. Radwan, A. M. Soliman, "Fractionalorder multi-phase oscillators design and analysis suitable for higherorder PSK applications," Analog Integrated Circuits and Signal Processing, vol. 87, no. 2, pp. 301-312, 2016.

[14] A. S. Elwakil, A. Agambayev, A. Allagui, K. N. Salama, "Experimental Demonstration of Fractional-Order Oscillators of Orders 2.6 and 2.7," Chaos, Solitons and Fractals, vol. 96, no. 3, pp. 160-164, 2017.

[15] R. Sotner, J. Jerabek, O. Domansky, N. Herencsar, A. Kartci and J. Dvořák, "Practical Design of Fractional-Order Oscillator Employing Simple Resonator and Negative Resistor," In Proc. of 2018 10th International Congress on Ultra Modern Telecommunications and Control Systems and Workshops (ICUMT), Moscow, Russia, 2018, pp. $1-4$.

[16] J. Koton, N. Herencsar, O. Sladok, J. W. Horng, "Pseudo-Differential Second-Order Band-Reject Filter Using Current Conveyors," AEU International Journal of Electronics and Communications, vol. 70, no. 6, pp. 814-821, 2016.

[17] D. Biolek, R. Senani, V. Biolkova, Z. Kolka, "Active elements for analog signal processing: Classification, Review and New Proposals," Radioengineering, vol. 17, no. 4, pp. 15-32, 2008.

[18] R. Senani, D. R. Bhaskar, and A. K. Singh, Current Conveyors: Variants, Applications and Hardware Implementations. Springer International Publishing, Switzerland, 2015.

[19] N. Herencsar, R. Sotner, B. Metin, J. Koton, K. Vrba, "New "Voltage Differencing' Device for Analog Signal Processing," In Proc. of the 8th International Conference on Electrical and Electronics Engineering (ELECO), Bursa, Turkey, 2013, pp. 17-20.

[20] J. Koton, N. Herencsar, K. Vrba, B. Metin, "Voltage-mode multifunction filter with mutually independent $\mathrm{Q}$ and w0 control feature using VDDDAs," Analog Integrated Circuits and Signal Processing, vol. 81, no. 1, pp. 53-60, 2014.

[21] R. Sotner, Z. Hrubos, N. Herencsar, J. Jerabek, T. Dostal, K. Vrba, "Precise Electronically Adjustable Oscillator Suitable for Quadrature Signal Generation Employing Active Elements with Current and Voltage Gain Control," Circuits, Systems, and Signal Processing, vol. 33, no. 1, pp. 1-35, 2014.

[22] J. Valsa, P. Dvorak, M. Friedl, "Network Model of CPE," Radioengineering, vol. 20, no. 3, pp. 619-626, 2011.

[23] J. Valsa, J. Vlach, "RC Models of a Constant Phase Element," Int. Journal of Circuit Theory and Applications, vol. 41, no. 1, pp. 59-67, 2013.

[24] O. Domansky, R. Sotner, L. Langhammer, J. Jerabek, C. Psychalinos, G. Tsirimokou, "Practical Design of RC Approximants of Constant Phase Elements and Their Implementation in Fractional-Order PID Regulators Using CMOS Voltage Differencing Current Conveyors," Circuits, Systems, and Signal Processing, vol. 38, no. 4, pp. 15201546, 2019.

[25] R. Sotner, J. Jerabek, A. Kartci, O. Domansky, N. Herencsar, V. Kledrowetz, B. B. Alagoz, C. Yeroglu, "Electronically Reconfigurable Two-Path Fractional-Order PI/D Controller Employing Constant Phase Blocks Based on Bilinear Segments Using CMOS Modified Current Differencing Unit," Microelectronics Journal, vol. 86, no. 4, pp. 114-129, 2019.

[26] G. Tsirimokou, C. Psychalinos, A. Elwakil and B. Maundy, "Fractional-Order Multiphase Sinusoidal Oscillator Design Using Current-Mirrors," In Proc. of 2018 41st International Conference on Telecommunications and Signal Processing (TSP), Athens, Greece, 2018, pp. 1-4. 